\title{
DISTINÇÃO DE GRUPOS ECOLÓGICOS DE ESPÉCIES FLORESTAIS POR MEIO DE TÉCNICAS MULTIVARIADAS ${ }^{1}$
}

\author{
José Humberto da Silva Santos², Rinaldo Luiz Caraciolo Ferreira ${ }^{3}$, José Antônio Aleixo da Silva ${ }^{3}$, \\ Agostinho Lopes de Souza ${ }^{4}$, Eufrázio de Souza Santos ${ }^{5}$, Isabelle Maria Jacqueline Meunier ${ }^{5}$
}

\begin{abstract}
RESUMO - Objetivou-se, neste estudo, distinguir grupos ecológicos por técnicas multivariadas, utilizando dados de 37 espécies arbóreas, em área sem intervenção, obtidos em 10 anos de monitoramento do Ensaio de Produção Sustentável em Floresta Estacional Semidecidual Secundária de Transição, implantado em 1986 em Rio Vermelho e Serra Azul de Minas, MG. As espécies foram divididas em pioneiras, secundárias iniciais e secundárias tardias. Já as variáveis consideradas foram: número de árvores por hectare; número de ingressos; número de árvores mortas; área basal; volume; diâmetro médio; incrementos em diâmetro, em área basal e em volume; índice de valor de importância; e regeneração natural. Utilizaram-se as análises de componentes principais (ACP), de agrupamento (AA) e discriminante (AD). Com a ACP foi possível reduzir a dimensão para tridimensional com uma explicação da variância de 79\%. Na AA, visando a uma classificação "a posteriori”, observou-se que a formação de grupos não correspondeu à classificação "a priori”. Com a AD, verificaram-se 92,86 e $57,14 \%$ de classificações "a posteriori" e "a priori” corretas, respectivamente. A utilização das ACP, AA e $\mathrm{AD}$ permitiu identificar as espécies arbóreas que deveriam ser classificadas em maior número de grupos ecológicos, enquanto a aplicação de técnicas multivariadas da avaliação da pré-classificação confirma a subjetividade na classificação dos grupos ecológicos.
\end{abstract}

Palavras-chave: Análise de agrupamento, componentes principais, discriminante, Floresta Estacional Semidecidual.

\section{DISTINCTION OF ECOLOGICAL GROUPS OF FOREST SPECIES THROUGH MULTIVARIATE TECHNIQUES}

\begin{abstract}
The objective of this research was to apply multivariete techniques analysis to separate ecological groups. Data of 37 tree species, in area without intervention, obtained in ten years of survey by the Experiment of Sustainable Production in Secondary Forest of Transition, established in 1986, at Rio Vermelho and Serra Azul de Minas, Minas Gerais State, Brazil were used. The species were separated in pioneers, early secondary and old secondary. The considered variables were: number of trees per hectare, number of ingrowth, mortality, basal area, volume, mean diameter, increment in diameter, increment in basal area, increment in volume, index of value of importance and natural regeneration. Principal components analysis (PCA); cluster analysis (CA) and the discriminant analysis (DA) were used. By PCA it was possible to reduce the dimension to threedimensional with variance explanation above $79 \%$. In the CA, seeking a classification at posteriori, it was observed that group formation did not correspond to the classification at priori. With the DA, 92.86 and $57.14 \%$ of classification at posteriori and at priori respectively were correct. In conclusion: the use of the principal components analysis, cluster analysis and discriminant analysis allowed the identification of tree species that should be classified in a larger number of ecological groups; and the application of PCA, CA and DA in the evaluation of at priori classification confirms most researchers' subjectivity in classifying ecological groups of tree species.
\end{abstract}

Key words: Cluster analysis, principal components analysis, discriminant analysis, semideciduos seasonal forest.

\footnotetext{
${ }^{1}$ Recebido para publicação em 09.1.2003 e aceito para publicação em 08.6.2004.

${ }^{2}$ Mestre em Biometria, Estatístico da Fundação de Saúde Amaury de Medeiros.<jhumbertosantos@bol.com.br>.

${ }_{3}^{3}$ Professor Adjunto da Universidade Federal Rural de Pernambuco e bolsista do CNPq. $<$ rinaldof@ufrpe.br $>$ e $<$ jaaleixo@uol.com.br>.

${ }^{4}$ Professor Titular da Universidade Federal de Viçosa e bolsista do CNPq. < <alsouza@ufv.br>.

${ }^{5}$ Professore Adjunto da Universidade Federal Rural de Pernambuco.<eufrazio@ufrpe.br>,<meunier@hotlink.com.br>.
} 


\section{INTRODUÇÃO}

As comunidades são unidades ecológicas muito complexas, e estudos dos ecossistemas são feitos com variáveis bióticas e abióticas, ligadas por uma complexa rede de inter-relações que rege o funcionamento desses ecossistemas. Por meio dessas interrelações são formados padrões estruturais, espaciais e temporais nas comunidades biológicas (PINTOCOELHO, 2000).

Em estudos ecológicos há tendência normal em agrupar amostras de características bióticas e, ou, abióticas ou associar espécies em comunidades com o objetivo do trabalho, buscando descrever, da maneira mais clara e sintética possível, a estrutura de um ecossistema, determinando a composição e a extensão das suas unidades funcionais. Pode-se, ainda, procurar ordenar amostras em função de um critério, visando simplificar, condensar e representar sinteticamente vastos conjuntos de dados, na expectativa de que as inter-relações ecológicas possam ser mais bem compreendidas. A necessidade do conhecimento dos padrões sucessionais de uma vegetação é um exemplo bem claro da tendência de simplificação das estruturas.

A classificação de espécies arbóreas em grupos sucessionais é comum, principalmente quanto às exigências de luz. As diversas classificações (BUDOWSKI, 1965; BAZZAZ, 1979; SWAINE e WHITMORE, 1988; LAMPRECHT, 1990; LEITÃO FILHO, 1993; JARDIM et al., 1996) se diferenciam quanto às denominações e às variáveis utilizadas. No entanto, tais classificações se baseiam na divisão das espécies florestais entre aquelas de estádios iniciais e tardios da sucessão.

A grande variedade de termos utilizados para distinguir grupos ecológicos de espécies em floresta tropical é, pelo menos, confusa e, algumas vezes, dificulta a comparação de estudos sobre regeneração natural e sucessão em diferentes florestas. Além disso, autores usam características distintas e, subjetivamente, montam seus sistemas de classificação. Logo, existe a necessidade de utilização de técnicas que possam retirar a subjetividade do pesquisador na classificação de grupos ecológicos de espécies arbóreas de florestas tropicais.

Considerando que as florestas são formadas por diversas espécies com os mais variados modos e formas de vida, uma análise envolvendo maior número de variáveis é ferramenta muito importante. Assim, a utilização de técnicas multivariadas é uma ferramenta útil, pois detectar, descrever e formular hipóteses sobre as possíveis inter-relações que regem os ecossistemas constituem os principais objetivos dessas hipóteses na área de ecologia.

A análise de dados multivariados ou multidimensionais baseia-se em matrizes de associação, das quais existem diversos tipos, cada um levando a resultados diferentes. Há dois caminhos alternativos para o estudo de vegetação: 1) estudos de análise de agrupamento, feita por algoritmos de aglomeração ou divisão, baseados em medidas de dissimilaridade; e 2) estudos de ordenação, usando a análise de componentes principais e a análise de correspondência (PIRESO'BRIEN e O'BRIEN, 1995).

A presente pesquisa objetivou distinguir grupos ecológicos de espécies florestais por técnicas multivariadas. Especificamente, procurou-se discriminar grupos ecológicos de espécies florestais por análises de componentes principais e de agrupamento e discriminantes, bem como verificar a pertinência de uma classificação a priori, por meio de análise discriminante.

\section{MATERIAL E MÉTODOS}

Foram utilizados dados do tratamento sem intervenção de 10 anos de monitoramento do Ensaio de Produção Sustentável em Floresta Secundária de Transição, implantado em 1986 pela Companhia Vale do Rio Doce. Detalhes do ensaio podem ser encontrados em Ferreira et al. (1999).

As espécies florestais foram divididas em três grupos ecológicos (FERREIRA, 1997), conforme o estádio sucessional (LEITÃO FILHO, 1993): 1. Pioneiras-Espécies que se desenvolvem em clareiras, nas bordas da floresta ou em locais abertos, sendo claramente dependentes de condições de maior luminosidade, não ocorrendo, em geral, no sub-bosque; 2 . Secundárias iniciais - Espécies que se desenvolvem em clareiras pequenas ou mais, raramente, no sub-bosque, em sombreamento, podendo também ocorrer em áreas de antigas clareiras, próximas às espécies pioneiras; e 3. Secundárias tardias - Espécies que se desenvolvem em sub-bosque permanentemente sombreado e, 
nesse caso, pequenas árvores ou espécies arbóreas de grande porte, que se desenvolvem lentamente em ambientes sombreados, podendo alcançar o dossel ou ser emergentes.
Foram consideradas 37 espécies com 10 ou mais indivíduos em 1986 (Quadro 1). Em cada ocasião foram estimados os números de árvores por hectare, de ingressos, de árvores mortas, de área basal, de vo-

Quadro 1 - Espécies arbóreas com mais de 10 indivíduos por grupo ecológico, ocorrentes na área experimental monitorada durante 10 anos em Rio Vermelho e Serra Azul de Minas Gerais, no Estado de Minas Gerais.

Table 1 - Tree species occurring with more than 10 individuals,per ecological group, occurring in the experimental area monitored for 10 years in Rio Vermelho and Serra Azul de Minas, Minas Gerais State, Brazil

\begin{tabular}{|c|c|c|c|}
\hline Código & Nome Vulgar Regional & Família/Espécie & Grupo Ecológico \\
\hline 20 & Gonçalo & $\begin{array}{l}\text { ANACARDIACEAE } \\
\text { Astronium fraxinifolium Schott } \\
\text { ANNONACEAE }\end{array}$ & Secundária inicial \\
\hline $\begin{array}{l}5 \\
32\end{array}$ & $\begin{array}{l}\text { Araticum } \\
\text { Pindaíba-Preta }\end{array}$ & $\begin{array}{l}\text { Annona cacans Warm. } \\
\text { Guatteria australis St. Hil. }\end{array}$ & $\begin{array}{l}\text { Secundária tardia } \\
\text { Secundária inicial }\end{array}$ \\
\hline 18 & Farinha-Seca & $\begin{array}{l}\text { AQUIFOLIACEAE } \\
\text { Ilex sp. }\end{array}$ & Secundária tardia \\
\hline 15 & Caroba, Perobinha, Carobinha & $\begin{array}{l}\text { BIGNONIACEAE } \\
\text { Jacaranda macrantha Cham. }\end{array}$ & Secundária inicial \\
\hline 21 & Imbiruçu-Bravo & $\begin{array}{l}\text { BOMBACACEAE } \\
\text { Eriotheca macrophylla (K. Schum.) A. Robyns }\end{array}$ & Secundária inicial \\
\hline $\begin{array}{l}10 \\
31 \\
6 \\
23\end{array}$ & $\begin{array}{l}\text { Canafístula } \\
\text { Pau-D’óleo } \\
\text { Braúna } \\
\text { Ingá-Burro }\end{array}$ & $\begin{array}{l}\text { LEGUMINOSAE CAESALPINIOIDAE } \\
\text { Cassia ferruginea Schrad. ex DC. } \\
\text { Copaifera langsdorffii Desf. } \\
\text { Melanoxylon brauna Schott } \\
\text { Sclerolobium sp. }\end{array}$ & $\begin{array}{l}\text { Secundária inicial } \\
\text { Secundária tardia } \\
\text { Secundária tardia } \\
\text { Secundária tardia }\end{array}$ \\
\hline 26 & Laranjinha & $\begin{array}{l}\text { CELASTRACEAE } \\
\text { Maytenus evonymoides Reiss. }\end{array}$ & Secundária inicial \\
\hline 17 & Coco-de-Cutia & $\begin{array}{l}\text { CHRYSOBALANACEAE } \\
\text { Licania sp. }\end{array}$ & Secundária inicial \\
\hline $\begin{array}{l}28 \\
14\end{array}$ & $\begin{array}{l}\text { Maria-Mole } \\
\text { Capoeirão }\end{array}$ & $\begin{array}{l}\text { COMPOSITAE } \\
\text { Piptocarpha macropoda Baker } \\
\text { Vernonia diffusa Less. }\end{array}$ & $\begin{array}{l}\text { Pioneira } \\
\text { Pioneira }\end{array}$ \\
\hline 13 & Canudo-de-Pito & $\begin{array}{l}\text { EUPHORBIACEAE } \\
\text { Mabea fistulifera Mart. }\end{array}$ & Pioneira \\
\hline $\begin{array}{l}7 \\
35\end{array}$ & $\begin{array}{l}\text { Cabiúna } \\
\text { Tira-Filho }\end{array}$ & $\begin{array}{l}\text { LEGUMINOSAE PAPILLIONOIDEAE } \\
\text { Dalbergia nigra (Vell) Fr. Allem. Ex Benth. } \\
\text { Machaerium nictitans Benth. }\end{array}$ & $\begin{array}{l}\text { Secundária inicial } \\
\text { Pioneira }\end{array}$ \\
\hline 36 & Uruvalheira & Machaerium triste Vog. & Secundária inicial \\
\hline $\begin{array}{l}8 \\
37\end{array}$ & $\begin{array}{l}\text { Calombo } \\
\text { Calombo-amarelo }\end{array}$ & $\begin{array}{l}\text { FLACOURTIACEAE } \\
\text { Casearia sp. } \\
\text { Xylosma sp. }\end{array}$ & $\begin{array}{l}\text { Secundária inicial } \\
\text { Secundária inicial }\end{array}$ \\
\hline 34 & Ruão & $\begin{array}{l}\text { GUTTIFERAE } \\
\text { Vismia ferruginea H.B.K. } \\
\text { LAURACEAE }\end{array}$ & Pioneira \\
\hline $\begin{array}{l}11 \\
12\end{array}$ & $\begin{array}{l}\text { Canela Amarela } \\
\text { Canela Prego, Sassafrás }\end{array}$ & $\begin{array}{l}\text { Nectandra oppositifolia Nees \& Mart. ex Nees } \\
\text { Ocotea odorifera Mez }\end{array}$ & $\begin{array}{l}\text { Secundária tardia } \\
\text { Secundária tardia }\end{array}$ \\
\hline
\end{tabular}


Quadro 1, cont.

Table 1, cont..

\begin{tabular}{|c|c|c|c|}
\hline Código & Nome Vulgar Regional & Família/Espécie & Grupo Ecológico \\
\hline & & MALPIGHIACEAE & \\
\hline 30 & Murici & Byrsonima sp. ${ }^{3}$ & Secundária inicial \\
\hline & & MELASTOMATACEAE & \\
\hline 33 & Roseira & Miconia cinnamomifolia Waudim & Pioneira \\
\hline & & LEGUMINOSAE MIMOSOIDEAE & \\
\hline 3 & Angico-Branco & Anadenanthera colubrina (Vell.) Brenan & Secundária inicial \\
\hline 24 & Ingá-Miúdo & Inga alba Willd. & Secundária inicial \\
\hline 22 & Ingá-Bananeira & Inga sp. & Secundária inicial \\
\hline 4 & Angiquinho & Mimosa pteridifolia Benth. & Secundária inicial \\
\hline 25 & Jacaré & Piptadenia gonoacantha Macbride & Pioneira \\
\hline 19 & Fruta-de-Pomba & $\begin{array}{l}\text { MYRTACEAE } \\
\text { Myrcia rostrata DC. }\end{array}$ & Secundária inicial \\
\hline & & OPILIACEAE & \\
\hline 27 & Limoeiro & Agonandra englerii Hoehne & Secundária tardia \\
\hline 29 & Marmelada & $\begin{array}{l}\text { RUBIACEAE } \\
\text { Psychotria carthagenensis Jacq. }\end{array}$ & Secundária inicial \\
\hline 9 & Cambuatã & $\begin{array}{l}\text { SAPINDACEAE } \\
\text { Cupania vernalis Cambess. }\end{array}$ & Secundária inicial \\
\hline 16 & Catuá & $\begin{array}{l}\text { SAPOTACEAE } \\
\text { Pouteria sp. }\end{array}$ & Secundária tardia \\
\hline 1 & Açoita-cavalo & $\begin{array}{l}\text { TILIACEAE } \\
\text { Luehea speciosa Willd. }\end{array}$ & Secundária tardia \\
\hline 2 & Alfavaca & $\begin{array}{l}\text { VERBENACEAE } \\
\text { Aloysia virgata Juss. }\end{array}$ & Secundária inicial \\
\hline
\end{tabular}

Adaptada de Ferreira (1997).

lume, de diâmetro médio, de incremento em diâmetro, em área basal e em volume, de índice de valor de importância e de regeneração natural. A partir dessas variáveis foram obtidas aquelas conferidas no Quadro 2.

Os grupos ecológicos de espécies foram discriminados em duas análises, sendo a primeira realizada sem considerar a divisão dos grupos (FERREIRA, 1997) e a segunda, levando-se em consideração a préclassificação realizada.

\subsection{Métodos estatísticos}

\subsubsection{Análise de componentes principais}

A análise de componentes principais foi aplicada visando à possibilidade de reduzir o conjunto original de variáveis.

Os dados originais foram padronizados com vistas a minimizar os efeitos das diferentes escalas de mensuração. A padronização foi realizada utilizando- se a média $X_{\mathrm{ij}}$ da j-ésima variável $(\mathrm{j}=1,2, \ldots ., 11)$ avaliada na i-ésima espécie $(i=1,2, \ldots, 107)$, gerando média zero e variância unitária (CRUZ e REGAZZI, 1997):

$$
\mathrm{Z}_{\mathrm{ij}}=\frac{\mathrm{X}_{\mathrm{ij}}-\overline{\mathrm{X}}_{\mathrm{i}}}{\mathrm{S}_{(\mathrm{Xi})}}
$$

Os componentes principais são combinações lineares de variáveis construídas de maneira a captar o máximo da variância, em que o primeiro componente explica a maior variação existente, o segundo a segunda maior variação, e assim sucessivamente. Diante dessa pressuposição, no estudo sobre classificação procurou-se estudar a dispersão das espécies em sistemas de eixos cartesianos onde a variabilidade disponível fosse maximizada. A técnica consiste na transformação de um conjunto de $\mathrm{n}$ variáveis padronizadas, $\mathrm{x}_{\mathrm{i} 1}$, $\mathrm{x}_{\mathrm{i} 2}, \ldots, \mathrm{x}_{\mathrm{in}}$ em um novo conjunto $\mathrm{y}_{\mathrm{i} 1}, \mathrm{y}_{\mathrm{i} 2}, \ldots, \mathrm{y}_{\mathrm{in}}, \mathrm{em}$ que os $\mathrm{y}_{\mathrm{i}^{\prime} \mathrm{s}}$ são funções lineares $\operatorname{dos} \mathrm{x}_{\mathrm{i}^{\prime} \mathrm{s}}$ e independentes entre si. As seguintes propriedades foram verificadas: 
Quadro 2 - Relação de variáveis utilizadas na distinção de grupos ecológicos de espécies florestais de técnicas multivariadas, em Rio Vermelho e Serra Azul de Minas, no Estado de Minas Gerais

Table 2 - List of variables used in the distinction of ecological groups of forest species through multivariate techniques, in Rio Vermelho and Serra Azul de Minas, Minas Gerais State, Brazil

\begin{tabular}{|c|c|c|}
\hline Código & Descrição & Unidade \\
\hline $\begin{array}{l}\text { DoA } 1, \text { DoA } 2, \text { DoA3, DoA4, } \\
\text { DoA5 e DoA6 }\end{array}$ & $\begin{array}{l}\text { Dominância absoluta em } 1986,1988,1990,1992, \\
1994 \text { e } 1996 \text {, respectivamente }\end{array}$ & $\mathrm{m}^{2} / \mathrm{ha}$ \\
\hline $\begin{array}{l}\text { DA } 1, \text { DA } 2, \text { DA } 3, \text { DA } 4, \\
\text { DA5 e DA6 }\end{array}$ & $\begin{array}{l}\text { Densidade absoluta em 1986, 1988, 1990, 1992, } \\
1994 \text { e } 1996 \text {, respectivamente }\end{array}$ & $\begin{array}{l}\text { Número de } \\
\text { árvores }\end{array}$ \\
\hline $\begin{array}{l}\text { FA } 1, \text { FA } 2, \text { FA } 3, \text { FA } 4 \\
\text { FA } 5 \text { e FA } 6\end{array}$ & $\begin{array}{l}\text { Freqüência absoluta em 1986, 1988, 1990, 1992, } \\
1994 \text { e 1996, respectivamente }\end{array}$ & $\%$ \\
\hline $\begin{array}{l}\text { IVC1, IVC2, IVC3, IVC4, } \\
\text { IVC5 e IVC6 }\end{array}$ & $\begin{array}{l}\text { Índice de valor de cobertura em 1986, 1988, 1990, } 1992 \text {, } \\
1994 \text { e } 1996 \text {, respectivamente }\end{array}$ & \\
\hline $\begin{array}{l}\text { IVI1, IVI2, IVI3, IVI4, } \\
\text { IVI5 e IVI6 }\end{array}$ & $\begin{array}{l}\text { Índice de valor de importância em 1986, 1988, 1990, } 1992 \text {, } \\
1994 \text { e 1996, respectivamente }\end{array}$ & \\
\hline $\begin{array}{l}\text { IP } 1, \text { IP } 2, \text { IP } 3, \text { IP } 4, \\
\text { IP5 e IPT }\end{array}$ & $\begin{array}{l}\text { Incremento periódico em diâmetro de } 86-88,88-90,90-92,92-94 \text {, } \\
94-96 \text { e } 86-96 \text {, respectivamente }\end{array}$ & $\mathrm{cm}$ \\
\hline $\begin{array}{l}\text { IG } 1, \text { IG } 2, \text { IG } 3, \text { IG } 4, \\
\text { IG5 e IGT }\end{array}$ & $\begin{array}{l}\text { Número de árvores ingressas } 86-88,88-90,90-92,92-94 \text {, } \\
94-96 \text { e } 86-96 \text {, respectivamente }\end{array}$ & $\begin{array}{l}\text { Número de } \\
\text { árvores }\end{array}$ \\
\hline T I & Taxa de ingresso & $\%$ \\
\hline $\begin{array}{l}\text { M2, M3, M4, } \\
\text { M5 е MT }\end{array}$ & $\begin{array}{l}\text { Número de árvores mortas } 88-90,90-92,92-94 \text {, } \\
94-96 \text { e } 86-96 \text {, respectivamente }\end{array}$ & $\begin{array}{l}\text { Número de } \\
\text { árvores }\end{array}$ \\
\hline TM & Taxa de mortalidade & $\%$ \\
\hline $\begin{array}{l}\text { IAB } 1, \text { IAB } 2, \text { IAB } 3, \text { IAB } 4, \\
\text { IAB } 5 \text { e IABT }\end{array}$ & $\begin{array}{l}\text { Incremento em área basal de } 86-88,88-90,90-92,92-94 \text {, } \\
94-96 \text { e } 86-96 \text {, respectivamente }\end{array}$ & $\mathrm{m}^{2} / \mathrm{ha}$ \\
\hline $\begin{array}{l}\mathrm{V} 1, \mathrm{~V} 2, \mathrm{~V} 3, \mathrm{~V} 4 \\
\text { V5 e V6 }\end{array}$ & $\begin{array}{l}\text { Volume em 1986, 1988, 1990, 1992, } \\
1994 \text { e } 1996 \text {, respectivamente }\end{array}$ & $\mathrm{m}^{3} / \mathrm{ha}$ \\
\hline $\begin{array}{l}\text { IV } 1, \text { IV } 2, \text { IV } 3, \text { IV4, } \\
\text { IV5 e IV6 }\end{array}$ & $\begin{array}{l}\text { Incremento em volume } 86-88,88-90,90-92,92-94 \text {, } \\
94-96 \text { e } 86-96 \text {, respectivamente }\end{array}$ & $\mathrm{m}^{3} / \mathrm{ha}$ \\
\hline $\begin{array}{l}\text { IGAB1, IGAB2, IGAB3, } \\
\text { IGAB4 e IGAB5 }\end{array}$ & $\begin{array}{l}\text { Área basal ingressa } 86-88,88-90,90-92, \\
92-94 \text { e } 94-96 \text {, respectivamente }\end{array}$ & $\mathrm{m}^{2} / \mathrm{ha}$ \\
\hline $\begin{array}{l}\text { MAB2, MAB3, } \\
\text { MAB4 e MAB5 }\end{array}$ & $\begin{array}{l}\text { Área basal das mortas } 88-90,90-92,92-94 \\
\text { e } 94-96 \text {, respectivamente }\end{array}$ & $\mathrm{m}^{2} / \mathrm{ha}$ \\
\hline $\begin{array}{l}\text { MV2, MV3, MV4 } \\
\text { e MV5 }\end{array}$ & $\begin{array}{l}\text { Volume das mortas } 88-90,90-92,92-94 \text { e } \\
94-96 \text {, respectivamente }\end{array}$ & $\mathrm{m}^{3} / \mathrm{ha}$ \\
\hline $\begin{array}{l}\text { IS } 1, \text { IS } 2, \text { IS } 3, \\
\text { IS } 4 \text { e IST }\end{array}$ & $\begin{array}{l}\text { Ingressos 86-88, 88-90, 90-92 e 92-94 sobreviventes em } 1996 \\
\text { e total de ingressos sobreviventes, respectivamente }\end{array}$ & $\begin{array}{l}\text { Número de } \\
\text { árvores }\end{array}$ \\
\hline $\begin{array}{l}\text { MI1, MI2, MI3, } \\
\text { MI4 e MIT }\end{array}$ & $\begin{array}{l}\text { Mortalidade de ingressos } 86-88,88-90,90-92, \\
92-94 \text { e } 86-96, \text { respectivamente }\end{array}$ & $\begin{array}{l}\text { Número de } \\
\text { árvores }\end{array}$ \\
\hline
\end{tabular}

a) Se $\mathrm{Y}_{\mathrm{ij}}$ é um componente principal, então:

$\mathrm{Y}_{\mathrm{ij}}=\mathrm{a}_{1} \mathrm{x}_{\mathrm{i} 1}+\mathrm{a}_{2} \mathrm{x}_{\mathrm{i} 2}+\ldots+\mathrm{a}_{\mathrm{n}} \mathrm{x}_{\mathrm{in}}$

b) $\mathrm{Se} \mathrm{Y}_{\mathrm{ij}}^{\prime}$ é outro componente principal, então:

$\mathrm{Y}_{\mathrm{ij}}^{\prime}=\mathrm{b}_{1} \mathrm{x}_{\mathrm{i} 1}+\mathrm{b}_{2} \mathrm{x}_{\mathrm{i} 2}+\ldots+\mathrm{b}_{\mathrm{n}} \mathrm{x}_{\mathrm{in}}$

$\sum_{j} a_{j}^{2}=\sum_{j} b_{j}^{2}=1 \sum_{j} a_{j} b_{j}=0$

ou seja, os componentes são independentes.

c) Os componentes principais foram obtidos pela solução do sistema: $\operatorname{det}\left(\mathrm{R}-\lambda_{1} \mathrm{I}\right) \mathrm{a}=0$, em que: $\mathrm{R}=$ matriz de correlação entre as médias estimadas, $\lambda_{1}=$ raízes características ou (autovalores) de $\mathrm{R}, \mathrm{I}=$ matriz identidade de dimensão pxp e a= vetor característico (ou autovetor) associado aos autovalores (CRUZ e REGAZZI, 1997). Dessa forma, os autovalores de R correspondem às variâncias de cada componente $\mathrm{e}$ os autovetores normalizados, aos coeficientes de ponderação dos caracteres padronizados.

A importância relativa de um componente foi calculada por: 
Importância de $Y_{j}=\frac{\lambda_{j}}{\operatorname{traço~}(R)}$

Após a determinação dos três primeiros componentes principais, que explicaram um mínimo de $80 \%$ da variação disponível, foram estimados os respectivos escores associados a cada espécie estudada.

\subsubsection{Análise de agrupamento}

A análise de agrupamento foi aplicada com base nos escores dos três primeiros componentes principais de cada espécie. Utilizou-se como medida de dissimilaridade a distância euclidiana estimada pela seguinte expressão:

$$
\mathrm{d}_{\mathrm{ii}}=\left[\sum_{\mathrm{j}}\left(\mathrm{cp}_{\mathrm{ij}}-\mathrm{cp}_{\mathrm{i}^{\prime} \mathrm{j}}\right)^{2}\right]^{1 / 2}
$$

em que: e i'; e

$\mathrm{d}_{\text {ii' }}=$ a distância euclidiana entre as espécies $\mathrm{i}$

$c p_{i j}-c p_{i^{\prime} j}=$ diferença entre escores dos componentes principais das espécies i e i', para uma variável j.

Para delimitação dos grupos, foi utilizado o método de Ward, conforme descrito por Souza et al. (1997).

\subsubsection{Análise discriminante}

A análise discriminante foi aplicada para verificar a pertinência dos grupos formados pela análise de agrupamento, de acordo com as funções discriminantes de Anderson (1984). Admitiu-se ainda, que a discriminação a ser feita envolve custos idênticos de má classificação e probabilidades conhecidas de várias populações.

Considerando que a função densidade de probabilidade associada à poulação pi é normal multivariada, então a função discriminante foi dada por:

$$
D_{i}\left(\begin{array}{c}
x \\
\sim
\end{array}\right)=-1 / 2 \ln \left[\sum_{i}\right]-1 / 2\left[\begin{array}{c}
\underset{\sim}{x}-\mu_{i} \\
\sim
\end{array}\right] \sum_{i}-1\left[\begin{array}{c}
x-\underset{\sim}{\mu_{i}} \\
\sim
\end{array}\right]+\ln \left(p_{i}\right)
$$

Pressupondo-se a igualdade das matrizes de covariâncias, então os componentes constantes de todo i podem ser retirados, e a função discriminante fica assim:

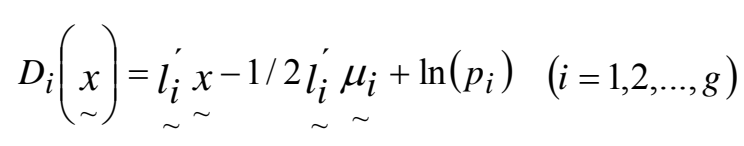

em que $\underset{\sim i}{l}=\sum^{-1} x_{i}$

A regra de decisão quanto à população de $\mathrm{x}$ foi a seguinte:

$D_{i}(\underset{\sim}{x})=\operatorname{máx}\left(D_{1}(\underset{\sim}{x}), D_{2}(\underset{\sim}{x}), \ldots, D_{g}(\underset{\sim}{x})\right)$.

Classificar $\mathrm{x}$ em $\pi \mathrm{i}$ se e somente se

$D_{i}(\underset{\sim}{x})=\stackrel{\wedge}{l_{i}^{\prime} x-1 / 2} \underset{\sim}{\stackrel{l_{i}^{\prime}}{x_{i}}+\ln \left(p_{i}\right)}$

Dessa forma, a partir das estimativas das matrizes de co-variância e de médias foram obtidas regras de discriminação, objetivando-se verificar a probabilidade de má classificação de cada grupo formado na análise de agrupamento.

As análises estatísticas foram efetuadas pelo Sistema SAS (Statistical Analysis System), versão 8.2 (SAS, 1999).

\section{RESULTADOS E DISCUSSÃO}

As estimativas dos coeficientes de variação, conforme cada grupo ecológico, foram altas em todas as variáveis, variando de 21,96 a 264,57, 10,80 a 364,19 e 19,42 a 316,23\%, respectivamente, em pioneiras, secundárias iniciais e secundárias tardias, o que dificulta a possibilidade de se criar uma única classificação para as espécies, pois a definição de grupo ecológico que melhor se ajusta a cada espécie deve ser muito criteriosa, uma vez que nem sempre existem limites naturais e claros entre os grupos (OLIVEIRA FILHO, 1994). A diferenciação entre as estratégias é gradual e, muitas vezes, inclui aspectos não-contemplados pelo sistema classificatório.

Foi possível a redução da octogésima nona para a terceira dimensão, com a acumulação de 79,45\% da variação total nos três primeiros componentes (Quadro 3). O grau de distorção sofrido na redução da dimensão foi de $20,55 \%$, considerado satisfatório se se levar em conta a redução da dimensão obtida. 
Quadro 3-Estimativas das variâncias (autovalores, $1_{\mathrm{j}}$ ) associadas aos componentes principais e suas importâncias relativas e acumuladas

Table 3 - Estimates of the variances (eigenvalues, $l_{\text {. }}$ ) associated to principal components and their relative and accumulated importances

\begin{tabular}{|c|c|c|c|}
\hline $\begin{array}{l}\text { Componentes } \\
\text { Principais }\end{array}$ & $\begin{array}{c}\text { Autovalor } \\
\left(\lambda_{\mathrm{j}}\right)\end{array}$ & $\begin{array}{c}\text { Proporção } \\
\text { da Variância } \\
(\%)\end{array}$ & $\begin{array}{r}\text { Proporção } \\
\text { Acumulada da } \\
\text { Variância (\%) }\end{array}$ \\
\hline 1 & 47,90 & 53,82 & 53,82 \\
\hline 2 & 14,00 & 15,73 & 69,55 \\
\hline 3 & 8,81 & 9,90 & 79,45 \\
\hline 4 & 5,75 & 6,46 & 85,91 \\
\hline 5 & 3,82 & 4,30 & 90,20 \\
\hline 6 & 1,82 & 2,04 & 92,25 \\
\hline 7 & 1,28 & 1,44 & 93,68 \\
\hline 8 & 0,94 & 1,06 & 94,74 \\
\hline 9 & 0,88 & 0,99 & 95,73 \\
\hline 10 & 0,74 & 0,84 & 96,57 \\
\hline 11 & 0,56 & 0,63 & 97,20 \\
\hline 12 & 0,49 & 0,55 & 97,75 \\
\hline 13 & 0,42 & 0,47 & 98,22 \\
\hline 14 & 0,31 & 0,35 & 98,57 \\
\hline 15 & 0,24 & 0,27 & 98,84 \\
\hline 16 & 0,21 & 0,23 & 99,08 \\
\hline 17 & 0,15 & 0,17 & 99,25 \\
\hline 18 & 0,15 & 0,17 & 99,42 \\
\hline 19 & 0,11 & 0,13 & 99,55 \\
\hline 20 & 0,10 & 0,11 & 99,66 \\
\hline 21 & 0,08 & 0,08 & 99,75 \\
\hline 22 & 0,05 & 0,06 & 99,81 \\
\hline 23 & 0,05 & 0,05 & 99,86 \\
\hline 24 & 0,03 & 0,04 & 99,90 \\
\hline 25 & 0,03 & 0,03 & 99,93 \\
\hline 26 & 0,02 & 0,02 & 99,95 \\
\hline 27 & 0,02 & 0,02 & 99,97 \\
\hline 28 & 0,01 & 0,01 & 99,98 \\
\hline 29 & 0,01 & 0,01 & 99,99 \\
\hline 30 & 0,00 & 0,00 & 100,00 \\
\hline 89 & 0,00 & 0,00 & 100,00 \\
\hline
\end{tabular}

Justifica-se a utilização das análises de componentes principais por proporcionarem uma simplificação estrutural dos dados originais. Os 29 primeiros componentes principais explicam $100 \%$ da variância, indicando que os 60 componentes principais restantes são combinações lineares dos 29 primeiros. O primeiro componente explica $53,82 \%$ da variância e os três primeiros, $79,45 \%$ da variação total, explicando satisfatoriamente a variabilidade manifestada entre os indivíduos avaliados, simplificando a interpretação.
Utilizando os escores referentes aos três primeiros componentes, realizaram-se as análises de agrupamento e discriminantes.

Com a aplicação da análise de agrupamento, considerando a formação de três grupos (Figura 1), observou-se que a formação de grupos (Quadro 4) não corresponde à classificação de Ferreira (1997), pois no Grupo 1 a espécie 31 é classificada como pioneira, enquanto a 86, secundária inicial; o Grupo 3 é formado por espécies pioneiras, secundárias iniciais e secundárias tardias, e apenas o Grupo 2 teria sua formação com a maioria das espécies de mesma classificação - secundária inicial. Vale salientar que as espécies que formam os Grupos 1 e 2 foram as espécies mais importantes da floresta, conforme Ferreira et al. (1999), e tiveram maior número de repetições e, conseqüentemente, melhor definição de comportamento.

Os termos de classificação: pioneira, secundária inicial, secundária tardia e clímax (BUDOWSKI, 1965); estádios iniciais e tardios da sucessão (BAZZAZ, 1979); pioneiras, nômades e tolerantes (MARTINEZ-RAMOS, 1985); lucíferas e umbrófilas (SWAINE e WHITMORE, 1988); heliófilas ou heliófitas, esciófilas e parcialmente esciófilas (LAMPRECHT, 1990); e pioneiras, secundária inicial e secundária tardia (LEITÃO FILHO, 1993) são uma grande simplificação teórica, e mais estudos deveriam ser realizados, principalmente quanto à natureza e ao número de variáveis inseridas na regra de classificação.

A pertinência dos três grupos obtidos com a análise de agrupamento foi verificada por meio de análise discriminante, verificando-se $92,86 \%$ de classificação correta (Quadro 5). Logo, esta seria a classificação recomendável na formação de três grupos ecológicos distintos das espécies arbóreas, levando-se em conta as variáveis consideradas.

Registraram-se apenas $57,14 \%$ de classificação correta, havendo a necessidade de relocação de 85,71 e $100 \%$ das espécies dos Grupos 1 (pioneiras) e 3 (secundárias tardias) para o Grupo 2 (secundárias iniciais), que apresentou $92 \%$ de espécies bem classificadas (Quadro 6). Isso está de acordo com Oliveira Filho (1994), segundo o qual o comportamento de espécies

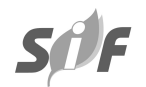

R. Árvore, Viçosa-MG, v.28, n.3, p. 387-396, 2004 


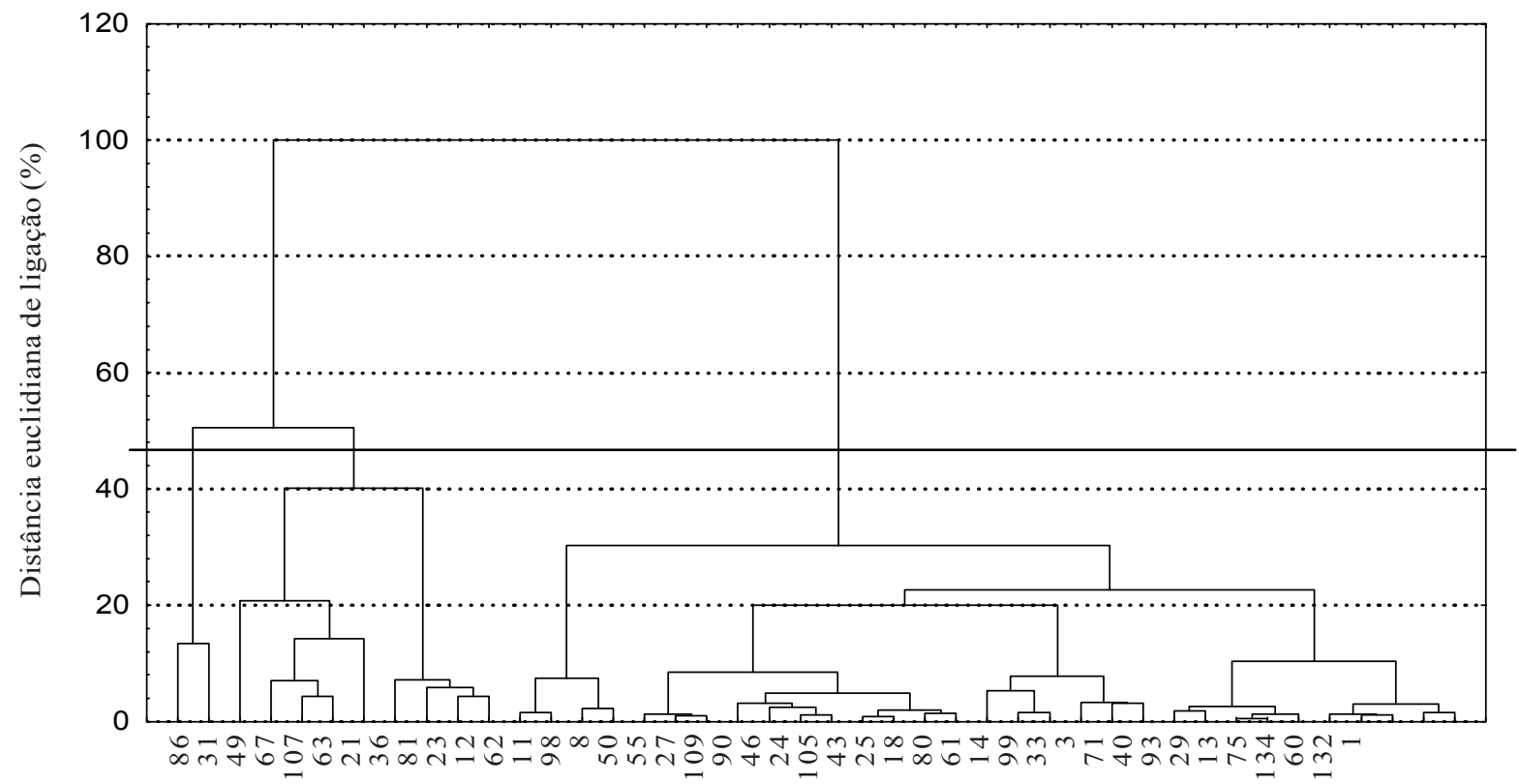

Figura 1 - Dendograma representando as seqüências de agrupamentos das 37 espécies, obtidas pelo método de Ward, com base na distância euclidiana.

Figure 1 - Dendogram representing the sequences of groupings of the 37 species, obtained by the method of Ward, basead on Euclidian distance.

Quadro 4-Grupos de dissimilaridade entre espécies a posteriori, estabelecidos pelo método de Ward a partir das distâncias euclidianas

Table 4 - Dissimilarity groups among species a posteriori through the method of Ward starting from Euclidian distance

\begin{tabular}{cl}
\hline Grupo & \multicolumn{1}{c}{ Espécie } \\
\hline 1 & 31 e 86 \\
2 & $12,21,23,36,49,63,67,81$ e 107 \\
3 & Demais espécies \\
\hline
\end{tabular}

pode variar de local para local. Portanto, são necessários estudos adicionais em mais de uma área para comprovar, aprimorar ou rejeitar generalizações sobre cada espécie em particular. Algumas espécies chegam a indicar comportamentos opostos em diferentes áreas, demonstrando que as estratégias das espécies são, via de regra, conjuntos interativos complexos de adaptações cujas propriedades emergentes podem
Quadro 5-Classificação correta, probabilidade de classificação e número de espécies em cada grupo classificados a posteriori, conforme análise discriminante

Table 5 - Correct classification, classification probability and number of species in each group classified a posteriori, according to discriminant analysis

\begin{tabular}{|c|c|c|c|c|}
\hline \multirow{2}{*}{$\begin{array}{c}\text { Grupo } \\
a\end{array}$} & \multirow{2}{*}{$\begin{array}{l}\text { Classificação } \\
\text { Correta (\%) }\end{array}$} & \multicolumn{3}{|c|}{ Grupo } \\
\hline & & 1 & 2 & 3 \\
\hline \multirow{2}{*}{\multicolumn{2}{|c|}{ posteriori }} & \multicolumn{3}{|c|}{$\begin{array}{c}\text { Probabilidade de } \\
\text { Classificação }\end{array}$} \\
\hline & & 0,738 & 0,214 & 0,048 \\
\hline 1 & 100,00 & 31 & 0 & 0 \\
\hline 2 & 66,67 & 3 & 6 & 0 \\
\hline 3 & 100,00 & 0 & 0 & 2 \\
\hline Total & 92,86 & 34 & 6 & 2 \\
\hline
\end{tabular}

extrapolar simplificações. Isso, possivelmente, explica os erros de classificação a priori dos Grupos 1 e 3, respectivamente. 
Quadro 6 - Classificação correta, probabilidade de classificação e número de espécies em cada grupo classificados a priori, conforme análise discriminante

Table 6 - Correct classification, classification probability and number of species in each group classified a priori, according to discriminant analysis

\begin{tabular}{lcccc}
\hline Grupo & Classificação & \multicolumn{3}{c}{ Grupo } \\
\cline { 3 - 5 } priori & Correta (\%) & & \multicolumn{3}{c}{$\begin{array}{c}\text { Probabilidade de } \\
\text { Classificação }\end{array}$} \\
\cline { 3 - 5 } & & 1 & 6,595 & 0,238 \\
\cline { 3 - 5 } & & 0,167 & 0,5 & 0 \\
\hline 1 & 14,29 & 1 & 23 & 0 \\
2 & 92,00 & 2 & 10 & 0 \\
3 & 0,00 & 0 & 39 & 0 \\
\hline
\end{tabular}

\section{CONCLUSÕES}

- A utilização das análises de componentes principais, de agrupamento e discriminantes permitiu identificar que as espécies arbóreas estudadas deveriam ser classificadas em maior número de grupos ecológicos.

- A aplicação de técnicas multivariadas na avaliação da pré-classificação confirmou a subjetividade da maioria dos pesquisadores na classificação de grupos ecológicos de espécies arbóreas.

\section{REFERÊNCIAS BIBLIOGRÁFICAS}

ANDERSON, T.W. An introduction to multivariate statistical analysis. New York: John Wiley \& Sons, 1984. 675 p.

BAZZAZ, F.A. The physiological ecology of plant sucession. Annual Review of Ecology and Systematics, v. 10, p. 351-371, 1979.

BUDOWSKI, G. Distribution of tropical american rain forest species in the light of sucessional processes. Turrialba, v. 15, n. 1, p. 40-42, 1965.

CRUZ, C.D.; REGAZZI, A.J. Modelos

biométricos aplicados ao melhoramento genético. Viçosa, MG: UFV, 1997. 390 p.
FERREIRA, R.L.C. Estrutura e dinâmica de uma floresta secundária de transição, Rio Vermelho e Serra Azul de Minas, MG. 1997. 208 f. Tese (Doutorado em Ciência Florestal) - Universidade Federal de Viçosa, Viçosa, 1997.

FERREIRA, R.L.C.; SOUZA, A.L.; SILVA, G.F. Dinâmica da estrutura de uma floresta secundária de transição. III - Estrutura horizontal. Revista Árvore, v. 23, n. 2, p. 157-168, 1999.

JARDIM, F.C.S.; SOUZA, A.L.; SILVA, A. F. Dinâmica da vegetação arbórea com DAP maior ou igual a 5,0 cm: comparação entre grupos funcionais e ecofisiológicos na estação experimental de silvicultura tropical do INPA, Manaus - AM. Revista Árvore, v. 20, n. 3, p. 267-278, 1996.

LAMPRECHT, H. Silvicultura nos trópicos: - Ecossistemas florestais e respectivas espécies arbóreas: possibilidades e métodos de aproveitamento sustentado. Eschborn: Instituto de Silvicultura da Universidade de Göttingen, GTZ, 1990. 343 p.

\section{LEITÃO FILHO, H.F. Ecologia da mata} atlântica em Cubatão. São Paulo: UNESP/ UNICAMP, 1993. 184 p.

MARTINEZ-RAMOS, M. Claros, ciclos vitales de los arboles tropicales y regeneracion natural de las selvas altas perennifolias. In: GOMEZPOMPA, A.; AMO, S.R. (Ed.). Investigaciones sobre la regeneracion de selvas altas en Vera Cruz, México. México: Editorial Alhambra Mexicana, 1985. T. 1, p. 191-239.

OLIVEIRA FILHO, A.T. Estudos ecológicos da vegetação como subsídio para programas de revegetação com espécies nativas: uma proposta metodológica. Cerne, v. 1. n. 1, p. 64-72, 1994.

PINTO-COELHO, R.M. Fundamentos em ecologia. Porto Alegre: Artes Médicas Sul, 2000. $252 \mathrm{p}$.

R. Árvore, Viçosa-MG, v.28, n.3, p. 387-396, 2004 
PIRES-O'BRIEN, M.J.; O'BRIEN, C.M. Ecologia e modelamento de florestas tropicais. Belém: FCAP/Serviço de Documentação e Informação, 1995.400 p.

SAS INSTITUTE. SAS OnlineDoc: version 8. Cary: 1999. CD-ROM.
SOUZA, A.L.; FERREIRA, R.L.C.; XAVIER, A. Análise de agrupamento aplicada à área florestal. Viçosa, MG: SIF, 1997. 109 p. (Documento SIF, 16).

SWAINE, M.D.; WHITMORE, T.C. On the definition of ecological species groups in tropical rain forests. Vegetatio, v. 75, p. 81-86, 1988. 